\title{
Insomnia Symptoms and Cardiovascular Disease among Older American Indians: The Native Elder Care Study
}

\author{
Charumathi Sabanayagam,, ${ }^{1,2}$ Anoop Shankar, ${ }^{1}$ Dedra Buchwald, ${ }^{3}$ and R. Turner Goins ${ }^{4}$ \\ ${ }^{1}$ Department of Community Medicine, West Virginia University School of Medicine, Robert C. Byrd Health Sciences Center, \\ 1 Medical Center Drive, P.O. Box 9190, Morgantown, WV 26505, USA \\ ${ }^{2}$ Singapore Eye Research Institute, Singapore National Eye Centre, 11 Third Hospital Avenue \#05-00, Singapore 168751 \\ ${ }^{3}$ Department of Medicine, University of Washington, Seattle, WA 98195, USA \\ ${ }^{4}$ Center for Healthy Aging Research, School of Social and Behavioral Health Sciences, College of Public Health and Human Sciences, \\ Oregon State University, Corvallis OR 97331, USA
}

Correspondence should be addressed to Charumathi Sabanayagam, csabanayagam@hsc.wvu.edu

Received 8 June 2011; Revised 8 September 2011; Accepted 22 September 2011

Academic Editor: Ivo Iavicoli

Copyright (C) 2011 Charumathi Sabanayagam et al. This is an open access article distributed under the Creative Commons Attribution License, which permits unrestricted use, distribution, and reproduction in any medium, provided the original work is properly cited.

Background. Cardiovascular disease (CVD) is the leading cause of death among American Indians. It is not known if symptoms of insomnia are associated with CVD in this population. Methods. We examined 449 American Indians aged $\geq 55$ years from the Native Elder Care Study. The main outcome-of-interest was self-reported CVD. Results. Short sleep duration, daytime sleepiness, and difficulty falling asleep were positively associated with CVD after adjusting for demographic, lifestyle, and clinical risk factors. Compared with a sleep duration of $7 \mathrm{~h}$, the multivariable odds ratio (OR) (95\% confidence interval [CI]) of CVD among those with sleep duration $\leq 5 \mathrm{~h}$ was 2.89 (1.17-7.16). Similarly, the multivariable OR (95\% CI) of CVD was 4.45 (1.85-10.72) and 2.60 (1.25-5.42) for daytime sleepiness $>2 \mathrm{~h}$ and difficulty falling asleep often/always. Conclusion. Symptoms of insomnia including short sleep duration, daytime sleepiness, and difficulty falling asleep are independently associated with CVD in American Indians aged $\geq 55$ years.

\section{Introduction}

Sleep complaints are common in older adults [1] and more than half of the older adults in a large population-based study had complaints related to inadequate sleep [2]. Recent studies have shown that sleep disturbances related to insomnia including short [3-5] or long $[4,5]$ sleep duration, daytime sleepiness [6], and difficulty falling asleep [3, 7] are associated with cardiovascular disease (CVD). Several prospective and cross-sectional studies have examined the association between sleep duration and CVD [3-5, 8-13]. However, there is limited information on the association between sleep duration and CVD in elderly populations [3, 9, 11]. Further, studies that examined the association between sleep disturbances including shorter or longer sleep duration, daytime sleepiness, difficulty falling asleep, and CVD among elderly adults were conducted in specific populations including women [9], men [14], non-Hispanic whites [3, 7, 11], or Japanese [11]. To our knowledge, no previous study has reported the association between insomnia symptoms and CVD in older American Indian adults. Although the multiracial Sleep Heart Health Study (SHHS) reported that sleep-disordered breathing was associated with heart failure, no subgroup analysis by race or ethnicity was provided in that report [15]. Among American Indians, CVD mortality rates equal or exceed national All Races rates [16] and CVD is the leading cause of death beginning at age 45 as opposed to age 65 in the general population [17]. Further, the prevalence of traditional CVD risk factors, including diabetes, hypertension, and dyslipidemia, is on the rise among American Indians [18]. However, it is not clear if insomnia symptoms contribute to the rising prevalence of CVD in this population. 
In this context, we examined the association between sleep duration, daytime sleepiness, and difficulty falling asleep and CVD in a population-based sample of American Indians.

\section{Methods}

The data for this study was collected as part of the Native Elder Care Study, a cross-sectional study of disability among older American Indians. Detailed selection of study population and methods has been reported previously $[19,20]$. In brief, a random sample of 633 American Indians aged $\geq 55$ years was drawn from a list of 1,430 individuals provided by tribal enrollment records. Of the 583 eligible participants, 505 participated in the baseline survey $(86.6 \%$ response rate). After excluding those with missing information on sleep variables $(n=14)$ and other variables included in the multivariable model $(n=42), 449$ subjects were included in the final analysis. Ethics approval was obtained from the tribe's institutional review board, Tribal Council, and Elder Council. This secondary data analysis was approved by the West Virginia University Institutional Review Board.

2.1. Assessment of CVD. The main outcome of interest was CVD defined as a physician diagnosis of heart attack, angina, heart failure, or stroke. This was determined by a "yes" response to the following four questions: "since age 55 has a doctor told you that you had angina, or heart attack, or congestive heart failure, or "stroke"?

2.2. Assessment of Exposure. Sleep duration, daytime sleepiness, and difficulty falling asleep were assessed from a questionnaire. Sleep duration for the 12-hour time period in night was assessed from the question "on average, how long do you sleep per night?" The response coded in hours was categorized into five groups for analysis: $\leq 5,6,7,8, \geq 9$ hours. In addition to night time sleep duration, we also assessed 24-hour sleep duration from the question, "how many total hours do you sleep in an average 24-hour period?" The response categorized into the same five groups as night time sleep duration. Daytime sleepiness for the 12-hour daytime period was assessed from the question "How often do you fall asleep during the day against your will [17]?" As almost everyone in this elderly cohort reported feeling sleepy at least for an hour during the daytime, the response was categorized into three groups: 1, 2, >2 hours. Difficulty falling asleep in the night was assessed from the question "How often do you have difficulty falling asleep or staying asleep?" The response categorized into 3 groups: never, sometimes, often/always.

Information on demographic, lifestyle, personal, and medical history was assessed from an interviewer-administered questionnaire. Education was recorded as the highest number of years of schooling completed and was categorized into below high school, high school, and above high school education. Current smokers were defined as those who answered affirmatively to the question "Do you currently smoke cigarettes?" Current alcohol drinking was defined as drinking alcohol everyday regardless of quantity. Physical activity was defined as engaging in physical activities such as running, swimming, aerobics, gardening, or walking for exercise. Symptoms of depression were assessed using the Center for Epidemiologic Studies depression Scale (CES-D) [21], a multidimensional screening instrument for major or clinical depression. In CES-D, each of the 20 response items is coded on a scale of $0-3$ with the total scores ranging from $0-60$. Depression was defined as a score of $\geq 16$ on the CES$D$ scale. Presence of diabetes mellitus, hypertension, cancer, chronic pain, and back pain were ascertained by a "yes" response to the following five questions: "Since age 55 has a doctor told that you had diabetes, or high blood pressure, or cancer, or back pain, or chronic pain syndrome? Height was measured in centimeters using a measuring tape and weight in kilograms using a weighing scale. Body mass index (BMI) was calculated as weight in kilograms divided by the square of height in meters.

2.3. Statistical Analysis. We first performed descriptive statistics for all variables. All analyses were performed incorporating the sampling weights to account for the oversampling of aged people. We then examined the association between categories of sleep duration ( $7 \mathrm{~h}$ as the reference), daytime sleepiness ( $1 \mathrm{~h}$ as the reference), and difficulty falling asleep (never as the reference) and CVD in two logistic regression models separately for each sleep disturbance: (1) adjusted for age and sex, (2) additionally adjusted for education (<high school, high school, above high school), current smoking (absent, present), current alcohol consumption (absent, present), physical activity (absent, present), BMI $\left(\mathrm{kg} / \mathrm{m}^{2}\right)$, diabetes (absent, present), hypertension (absent, present), depression (absent, present), cancer (absent, present), chronic pain (absent, present), and back pain (absent, present). As the three sleep disturbances might be intercorrelated with each other, in a separate analysis, we included all three sleep variables simultaneously in the multivariable model and repeated the analysis. We performed tests for trend using the categories of sleep variables as ordinal variables in the corresponding multivariable model. All statistical analyses were performed using SAS version 9.1.

\section{Results}

The mean age of the study population was $65.9 \mathrm{y}(\mathrm{SD}=$ $8.5 \mathrm{y}$ ) and $62 \%$ were female. The prevalence of various comorbidities was high in this elderly population (diabetes $=40.9 \%$, hypertension $=56.6 \%$, depression $=13.8 \%$, cancer $=9.5 \%$, chronic pain $=11.2 \%$, and back pain $=34.9 \%$ ) (data not shown). Table 1 shows the baseline characteristics of the study population by gender. Women were less likely to be current drinkers and more likely to report difficulty falling asleep than men. There was no significant difference $(P=0.9)$ in the reported average sleep duration between men and women $(6.8$ versus $6.9 \mathrm{~h})$. The prevalence of each sleep disturbance is presented in Figure 1. $21.3 \%$ of the participants reported sleeping $7 \mathrm{~h}$ and $14.8 \%$ of the participants reported sleeping $\leq 5$ h. $6.7 \%$ of the participants reported having daytime sleepiness $>2 \mathrm{~h}$ and $16.6 \%$ of the participants had difficulty falling asleep often/always. 
TABLE 1: Baseline characteristics of the study population by gender.

\begin{tabular}{|c|c|c|c|}
\hline Characteristics & $\operatorname{Men}(n=163)$ & Women $(n=286)$ & $P$-value \\
\hline Age (years) & $64.9(8.0)$ & $66.4(8.7)$ & 0.06 \\
\hline Education categories (\%) & & & 0.5 \\
\hline Below high school & 29.1 & 33.2 & \\
\hline High school & 30.9 & 32.2 & \\
\hline Above high school & 40.0 & 34.6 & \\
\hline Current smoker (\%) & 21.8 & 27.7 & 0.2 \\
\hline Current drinker (\%) & 19.6 & 8.1 & 0.0003 \\
\hline Moderate physical activity (\%) & 66.8 & 57.6 & 0.05 \\
\hline Body mass index $\left(\mathrm{kg} / \mathrm{m}^{2}\right)$ & $30.0(5.4)$ & $29.9(6.7)$ & 0.9 \\
\hline Diabetes mellitus (\%) & 36.8 & 43.5 & 0.2 \\
\hline Hypertension (\%) & 54.8 & 57.7 & 0.5 \\
\hline Cancer $(\%)$ & 10.6 & 15.9 & 0.1 \\
\hline Depression (\%) & 8.2 & 10.4 & 0.4 \\
\hline Chronic pain (\%) & 11.4 & 11.1 & 0.9 \\
\hline Back pain $(\%)$ & 30.3 & 37.7 & 0.1 \\
\hline Night sleep duration (hours) & $6.8(1.7)$ & $6.9(1.6)$ & 0.9 \\
\hline Daytime sleepiness (hours) & $1.6(0.7)$ & $1.4(0.6)$ & 0.05 \\
\hline Difficulty falling asleep (hours) & $1.7(0.8)$ & $1.9(0.9)$ & 0.003 \\
\hline
\end{tabular}

* Data presented are percentages or mean values (standard deviation), as appropriate for the variable.

TABLE 2: Association between sleep variables and cardiovascular disease.

\begin{tabular}{|c|c|c|c|c|}
\hline Sleep variables & No at risk* $(n=449)$ & $\begin{array}{c}\text { Weighted prevalence } \\
\text { of CVD, \% }\end{array}$ & $\begin{array}{l}\text { Age, sex adjusted } \\
\text { OR }(95 \% \text { CI })\end{array}$ & $\begin{array}{c}\text { Multivariable adjusted } \\
\text { OR }(95 \% \mathrm{CI})^{\dagger}\end{array}$ \\
\hline \multicolumn{5}{|l|}{ Sleep duration, $\mathrm{h}$} \\
\hline$\leq 5$ & 58 & 29.2 & $3.51(1.55-7.95)$ & $2.89(1.17-7.16)$ \\
\hline 6 & 101 & 17.6 & $1.55(0.71-3.42)$ & $1.32(0.56-3.09)$ \\
\hline 7 & 98 & 12.6 & 1 (referent) & 1 (referent) \\
\hline 8 & 145 & 22.1 & $1.85(0.89-3.85)$ & $1.67(0.76-3.66)$ \\
\hline$\geq 9$ & 47 & 18.5 & $1.46(0.54-3.93)$ & $1.15(0.38-3.53)$ \\
\hline \multicolumn{5}{|c|}{ Frequency of daytime sleepiness, $\mathrm{h}$} \\
\hline 1 & 253 & 14.3 & 1 (referent) & 1 (referent) \\
\hline 2 & 161 & 22.6 & $1.53(0.91-2.57)$ & $1.33(0.76-2.34)$ \\
\hline$>2$ & 35 & 54.6 & $6.06(2.71-13.52)$ & $4.45(1.85-10.72)$ \\
\hline$P$-trend & & & $<0.0001$ & 0.003 \\
\hline \multicolumn{5}{|c|}{ Difficulty falling asleep } \\
\hline Never & 200 & 14.8 & 1 (referent) & 1 (referent) \\
\hline Sometimes & 178 & 20.0 & $1.60(0.92-2.78)$ & $1.23(0.68-2.25)$ \\
\hline Often or always & 71 & 31.9 & $3.39(1.76-6.53)$ & $2.60(1.25-5.42)$ \\
\hline$P$-trend & & & 0.0004 & 0.02 \\
\hline
\end{tabular}

Abbreviations: CI: confidence interval; OR: odds ratio.

* Unweighted sample size

${ }^{\dagger}$ Adjusted for age (years), sex (men, women), education (<high school, high school, >high school), current smoking (absent, present), current alcohol consumption (absent, present), physical activity (absent, present), body mass index $\left(\mathrm{kg} / \mathrm{m}^{2}\right)$, diabetes (absent, present), hypertension (absent, present), depression (absent, present), cancer (absent, present), chronic pain (absent, present) and back pain (absent, present).

In univariate analysis, older age, below high school education, diabetes, hypertension, cancer, chronic pain, and back pain were associated with CVD; older age, current smoking, depression, chronic pain, and back pain were significantly associated with sleep duration $\leq 5 \mathrm{~h}($ all $P<0.05)$.
Table 2 shows the relationship between the sleep disturbances and CVD after adjusting for potential confounders. The prevalence of CVD was highest among those who reported sleeping $\leq 5 \mathrm{~h}$ and lowest among those who reported sleeping $7 \mathrm{~h}$. There was a significant positive association 


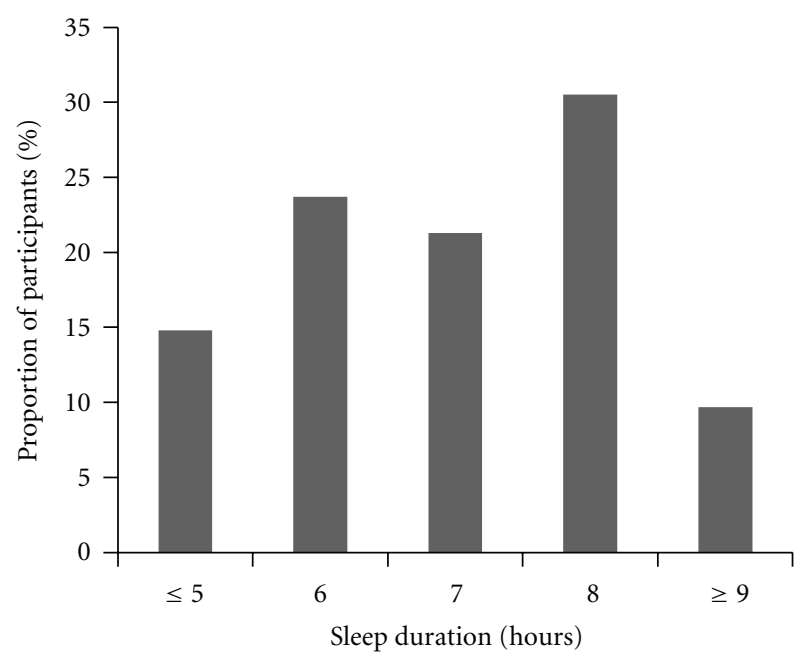

(a)

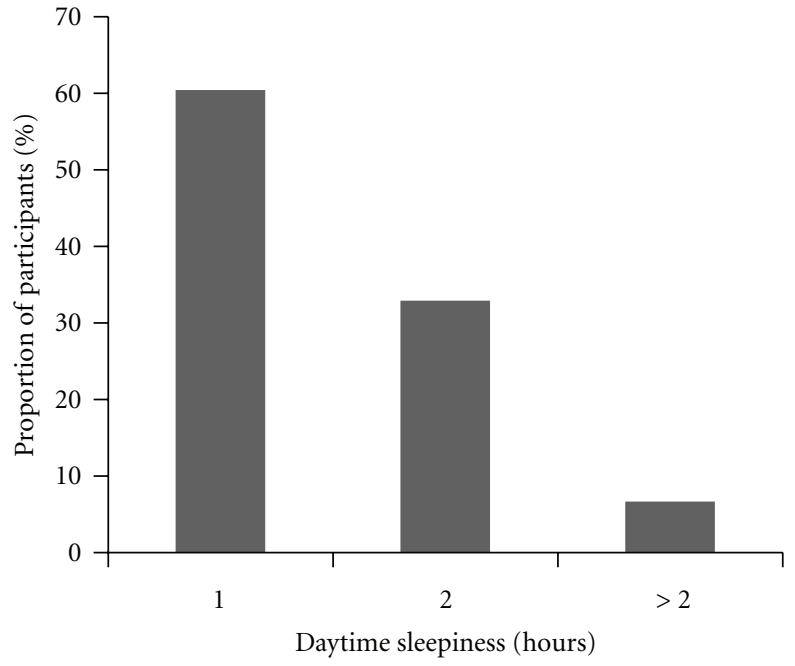

(b)

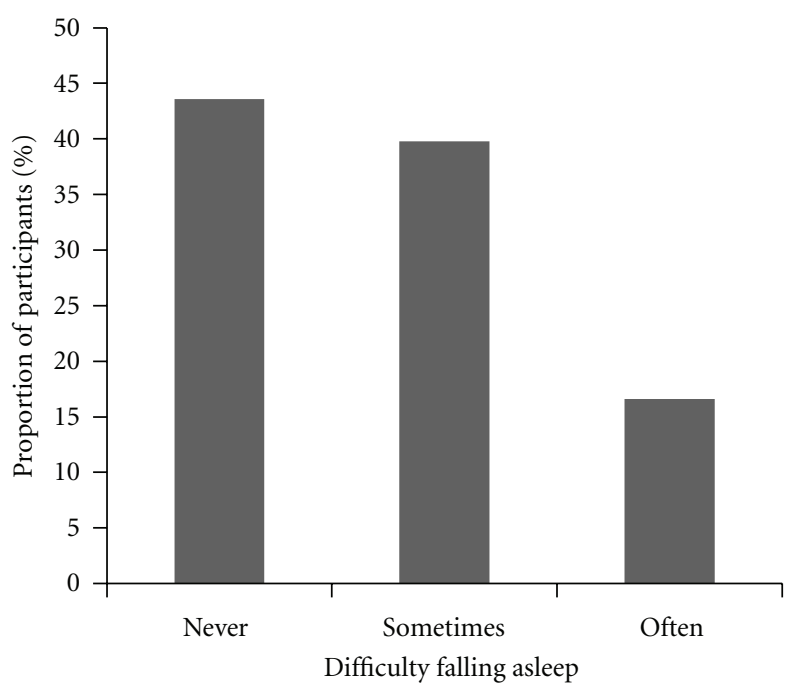

(c)

Figure 1: Prevalence of sleep disturbances in the study population.

between sleep duration $\leq 5 \mathrm{~h}$ and CVD in both the age, sexadjusted, and the multivariable model additionally adjusted for education, current smoking, current alcohol drinking, physical activity, diabetes mellitus, hypertension, depression, cancer, chronic pain, and back pain. The prevalence of CVD increased with increasing categories of daytime sleepiness $(P$-trend $<0.0001)$ and daytime sleepiness $>2 \mathrm{~h}$ was significantly associated with CVD in both the models. Similarly, the prevalence of CVD increased with increasing categories of difficulty falling asleep $(P$-trend $=0.006)$ and the association between difficulty falling asleep often/always was significant in both the models.

In a supplementary analysis, when we included all three sleep disturbances simultaneously in the multivariable model, although all 3 sleep disturbances showed a positive magnitude of association with CVD, only the association of daytime sleepiness $>2 \mathrm{~h}$ with CVD was statistically significant
$($ OR $[95 \%]=4.16[1.67-10.32]$ for daytime sleepiness $>2 \mathrm{~h}$ ) and not for sleep duration or difficulty falling asleep (OR $[95 \%]=1.90[0.68-5.34]$ for sleep duration $\leq 5 \mathrm{~h}$ and 2.00 [0.84-4.76] for difficulty falling asleep). In a second supplementary analysis, when we assessed the association between $24 \mathrm{~h}$ sleep duration and CVD, the pattern of association was similar to that of night time sleep duration. Compared with a sleep duration of $7 \mathrm{~h}$, the multivariable odds ratio (OR) $(95 \%$ confidence interval [CI]) of CVD was 5.37 (1.94-14.91) for sleep duration $\leq 5 \mathrm{~h}$ and $2.26(0.92-5.54)$ for $>8 \mathrm{~h}$.

\section{Discussion}

In a population-based study of American Indian adults aged $\geq 55$ years, short sleep duration, daytime sleepiness, and difficulty falling asleep were significantly associated with CVD independent of age, sex, education, current 
smoking, current alcohol consumption, physical activity, BMI, diabetes, hypertension, depression, cancer, chronic pain, and back pain. To our knowledge, this is the first study to separately report the association between various sleep characteristics and CVD among American Indians. Our findings of associations between sleep duration, daytime sleepiness, difficulty falling asleep, and CVD are consistent with previous studies that reported a similar association in older Hispanic and non-Hispanic whites and African Americans $[3,4,6,7]$ and extend the association to middleaged and older adults in the American Indian population.

In the current study, sleep duration $\leq 5 \mathrm{~h}$ was associated with CVD. Similar to our report, sleep duration $<6 \mathrm{~h}$ was associated with CVD among 1506 men and women aged $\geq 55$ years who participated in the 2003 Sleep in America poll [3] and sleep duration $<7.5 \mathrm{~h}$ was associated with incident CVD among 1255 elderly adults with hypertension in Japan [13]. Few studies have documented a " $U$ " shaped association between sleep duration and CVD $[4,5,8]$ and CVD mortality $[22,23]$. A recent meta-analysis including 15 prospective studies documented a positive association between both short and long durations of sleep and CVD [8]. Both short and long durations of sleep were associated with CVD among adults aged $>60$ years in the National Health Interview Survey 2005 [4] and with coronary heart disease among female health professionals aged 45-65 years in the Nurses' Health study [5]. In contrast to our study, few studies have documented an association between long duration of sleep and CVD $[9,12]$. Sleep duration $\geq 9$ hours was shown to be associated with CVD among a large cohort of postmenopausal women in the Women's Health Initiative [9] and 3,430 middle-aged Chinese adults in Taiwan [12].

In the current study, daytime sleepiness and difficulty falling asleep were associated with CVD similar to previous reports. In the Cardiovascular Health Study including 5,888 whites and African Americans [6], daytime sleepiness was associated with CVD, and in an earlier study, difficulty falling asleep was associated with angina in the same population [7]. Daytime sleepiness was associated with stroke among 7,844 adults who participated in the NHANES-I followup study in the US and a large cohort of men aged 55-69 years in the UK [14].

An association between symptoms of insomnia and CVD is biologically plausible. Short sleep duration, daytime sleepiness, and difficulty falling asleep through metabolic and endocrine dysfunctions lead to impaired glucose tolerance, increased insulin resistance, enhanced sympathetic activity, and increased blood pressure with resultant CVD [24, 25]. Studies have also documented an association between insomnia symptoms and risk factors of CVD including diabetes $[3,26]$, hypertension [27], obesity [28] dyslipidemia [29], and metabolic syndrome [30].

The strengths of the current study include its populationbased nature and the availability of data on potential confounders. Our study has several limitations. First, sleep habits were self-reported and the associated measurement error might have resulted in a nondifferential misclassification of exposure. Second, we assessed daytime sleepiness using a single question rather than the standard Epworth Sleepiness
Score (ESS). However, daytime sleepiness assessed using a single question is comparable to that measured using ESS [31] and several epidemiological studies have assessed daytime sleepiness using a single question similar to ours [32, 33]. Third, it is possible that our results may be biased by residual confounding from unmeasured variables including sleep disordered breathing (SDB) that play a role in CVD. Fourth, although the sample size was adequate to detect an overall association, it was insufficient to perform subgroup analysis and to explore effect modifications. Fifth, because of the cross-sectional nature of the study, we cannot infer causal associations.

In conclusion, our study shows that short sleep duration, daytime sleepiness, and difficulty falling asleep were associated with CVD among American Indians. If confirmed by future prospective studies, our findings may have important implications for targeting sleep habits in intervention programs intended to reduce the burden of CVD among American Indians.

\section{Conflict of Interests}

There are no conflicts of interest related to this manuscript.

\section{Disclosure}

The guarantor, C. Sabanayagam, accepts full responsibility for the work and/or the conduct of the study. She had access to the data and controlled the decision to publish.

\section{Acknowledgments}

The National Institute on Aging at National Institutes of Health (K01 AG022336 to R. T. Goins) and from the American Heart Association National Clinical Research Program Grant (to A. Shankar) are acknowledged.

\section{References}

[1] P. N. Prinz, M. V. Vitiello, M. A. Raskind, and M. J. Thorpy, "Geriatrics: sleep disorders and aging," New England Journal of Medicine, vol. 323, no. 8, pp. 520-526, 1990.

[2] D. J. Foley, A. A. Monjan, S. L. Brown, E. M. Simonsick, R. B. Wallace, and D. G. Blazer, "Sleep complaints among elderly persons: an epidemiologic study of three communities," Sleep, vol. 18, no. 6, pp. 425-432, 1995.

[3] D. Foley, S. Ancoli-Israel, P. Britz, and J. Walsh, "Sleep disturbances and chronic disease in older adults: results of the 2003 National Sleep Foundation Sleep in America Survey," Journal of Psychosomatic Research, vol. 56, no. 5, pp. 497-502, 2004.

[4] C. Sabanayagam and A. Shankar, "Sleep duration and cardiovascular disease: results from the National Health Interview Survey," Sleep, vol. 33, no. 8, pp. 1037-1042, 2010.

[5] N. T. Ayas, D. P. White, J. E. Manson et al., "A prospective study of sleep duration and coronary heart disease in women," Archives of Internal Medicine, vol. 163, no. 2, pp. 205-209, 2003.

[6] A. B. Newman, C. F. Spiekerman, P. Enright et al., "Daytime sleepiness predicts mortality and cardiovascular disease in older adults," Journal of the American Geriatrics Society, vol. 48, no. 2, pp. 115-123, 2000. 
[7] A. B. Newman, P. L. Enright, T. A. Manolio, E. F. Haponik, and P. W. Wahl, "Sleep disturbance, psychosocial correlates, and cardiovascular disease in 5201 older adults: the Cardiovascular Health Study," Journal of the American Geriatrics Society, vol. 45, no. 1, pp. 1-7, 1997.

[8] F. P. Cappuccio, D. Cooper, L. Delia, P. Strazzullo, and M. A. Miller, "Sleep duration predicts cardiovascular outcomes: a systematic review and meta-analysis of prospective studies," European Heart Journal, vol. 32, no. 12, pp. 1484-1492, 2011.

[9] J. C. Chen, R. L. Brunner, H. Ren et al., "Sleep duration and risk of ischemic stroke in postmenopausal women," Stroke, vol. 39, no. 12, pp. 3185-3192, 2008.

[10] A. I. Qureshi, W. H. Giles, J. B. Croft, and D. L. Bliwise, "Habitual sleep patterns and risk for stroke and coronary heart disease: a 10-year follow-up from NHANES I," Neurology, vol. 48, no. 4, pp. 904-911, 1997.

[11] E. Suzuki, T. Yorifuji, K. Ueshima et al., "Sleep duration, sleep quality and cardiovascular disease mortality among the elderly: a population-based cohort study," Preventive Medicine, vol. 49, no. 2-3, pp. 135-141, 2009.

[12] K. L. Chien, P. C. Chen, H. C. Hsu et al., "Habitual sleep duration and insomnia and the risk of cardiovascular events and all-cause death: report from a community-based cohort," Sleep, vol. 33, no. 2, pp. 177-184, 2010.

[13] K. Eguchi, T. G. Pickering, J. E. Schwartz et al., "Short sleep duration as an independent predictor of cardiovascular events in Japanese patients with hypertension," Archives of Internal Medicine, vol. 168, no. 20, pp. 2225-2231, 2008.

[14] P. Elwood, M. Hack, J. Pickering, J. Hughes, and J. Gallacher, "Sleep disturbance, stroke, and heart disease events: evidence from the Caerphilly cohort," Journal of Epidemiology and Community Health, vol. 60, no. 1, pp. 69-73, 2006.

[15] D. J. Gottlieb, G. Yenokyan, A. B. Newman et al., "Prospective study of obstructive sleep apnea and incident coronary heart disease and heart failure: the sleep heart health study," Circulation, vol. 122, no. 4, pp. 352-360, 2010.

[16] Indian Health service, Trends in Indian Health, 2002-2003, US Department of Health and Human Services, 2009.

[17] Indian Health service, IHS Chart Series Book, US Department of Health and Human Services, 1984.

[18] T. K. Welty, D. A. Rhoades, F. Yeh et al., "Changes in cardiovascular disease risk factors among American Indians: the Strong Heart Study," Annals of Epidemiology, vol. 12, no. 2, pp. 97106, 2002.

[19] R. T. Goins and C. S. Pilkerton, "Comorbidity among older American Indians: the Native Elder Care Study," Journal of Cross-Cultural Gerontology, vol. 25, no. 4, pp. 343-354, 2010.

[20] R. T. Goins, S. M. Spencer, S. Goli, and J. C. Rogers, "Assistive technology use of older american indians in a southeastern tribe: the native elder care study," Journal of the American Geriatrics Society, vol. 58, no. 11, pp. 2185-2190, 2010.

[21] L. S. Radloff, "The CES-D scale: a self-report depression scale for research in the general population," Applied Psychological Measures, vol. 1, pp. 385-401, 1977.

[22] A. Shankar, W. P. Koh, J. M. Yuan, H. P. Lee, and M. C. Yu, "Sleep duration and coronary heart disease mortality among Chinese adults in Singapore: a population-based cohort study, American Journal of Epidemiology, vol. 168, no. 12, pp. 1367-1373, 2008.

[23] S. Ikehara, H. Iso, C. Date et al., "Association of sleep duration with mortality from cardiovascular disease and other causes for japanese men and women: the JACC study," Sleep, vol. 32, no. 3, pp. 295-301, 2009.
[24] K. Spiegel, R. Leproult, and E. Van Cauter, "Impact of sleep debt on metabolic and endocrine function," Lancet, vol. 354, no. 9188, pp. 1435-1439, 1999.

[25] E. Van Cauter, U. Holmbäck, K. Knutson et al., "Impact of sleep and sleep loss on neuroendocrine and metabolic function," Hormone Research, vol. 67, no. 1, pp. 2-9, 2007.

[26] E. R. Chasens, "Obstructive sleep apnea, daytime sleepiness, and type 2 diabetes," Diabetes Educator, vol. 33, no. 3, pp. 475482, 2007.

[27] J. E. Gangwisch, S. B. Heymsfield, B. Boden-Albala et al., "Short sleep duration as a risk factor for hypertension: analyses of the first National Health and Nutrition Examination Survey," Hypertension, vol. 47, no. 5, pp. 833-839, 2006.

[28] S. R. Patel and F. B. Hu, "Short sleep duration and weight gain: a systematic review," Obesity, vol. 16, no. 3, pp. 643-653, 2008.

[29] Y. Kaneita, M. Uchiyama, N. Yoshiike, and T. Ohida, "Associations of usual sleep duration with serum lipid and lipoprotein levels," Sleep, vol. 31, no. 5, pp. 645-652, 2008.

[30] W. M. Troxel, D. J. Buysse, K. A. Matthews et al., "Sleep symptoms predict the development of the metabolic syndrome," Sleep, vol. 33, no. 12, pp. 1633-1640, 2010.

[31] S. N. Zallek, R. Redenius, H. Fisk, C. Murphy, and E. O’Neill, "A single question as a sleepiness screening tool," Journal of Clinical Sleep Medicine, vol. 4, no. 2, pp. 143-148, 2008.

[32] N. F. Watson, J. Goldberg, L. Arguelles, and D. Buchwald, "Genetic and environmental influences on insomnia, daytime sleepiness, and obesity in twins," Sleep, vol. 29, no. 5, pp. 645649, 2006.

[33] E. V. A. Lindberg, N. E. D. Carter, T. Gislason, and C. Janson, "Role of snoring and daytime sleepiness in occupational accidents," American Journal of Respiratory and Critical Care Medicine, vol. 164, no. 11, pp. 2031-2035, 2001. 


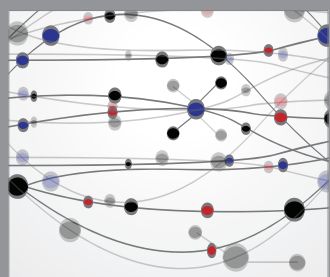

The Scientific World Journal
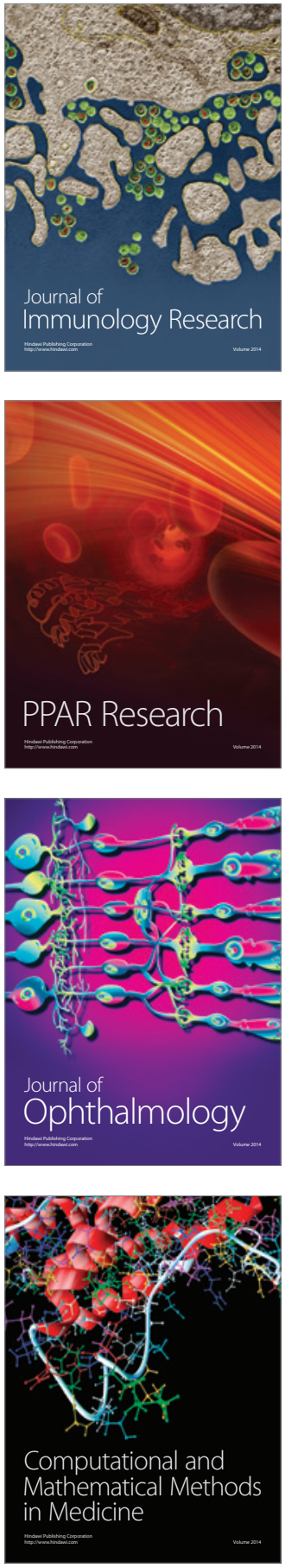

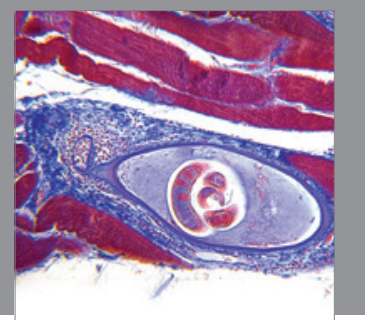

Gastroenterology

Research and Practice
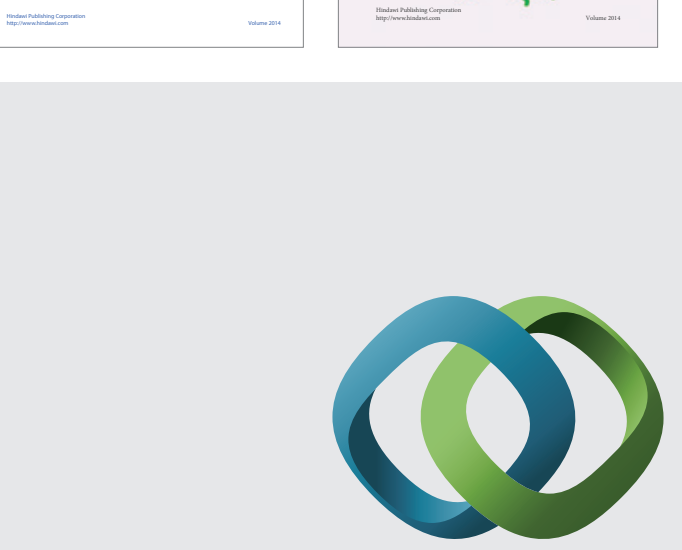

\section{Hindawi}

Submit your manuscripts at

http://www.hindawi.com
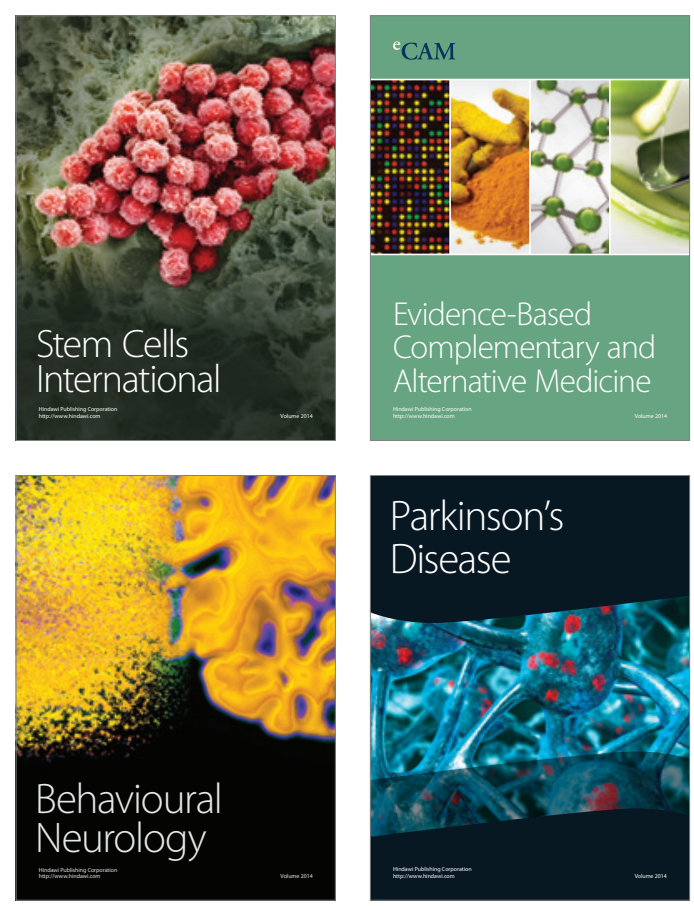

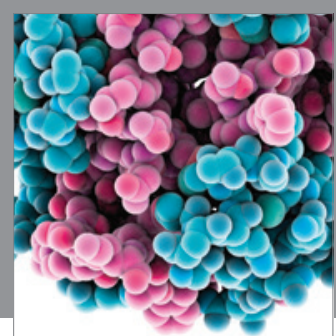

Journal of
Diabetes Research

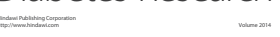

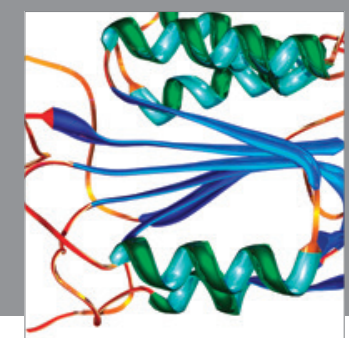

Disease Markers
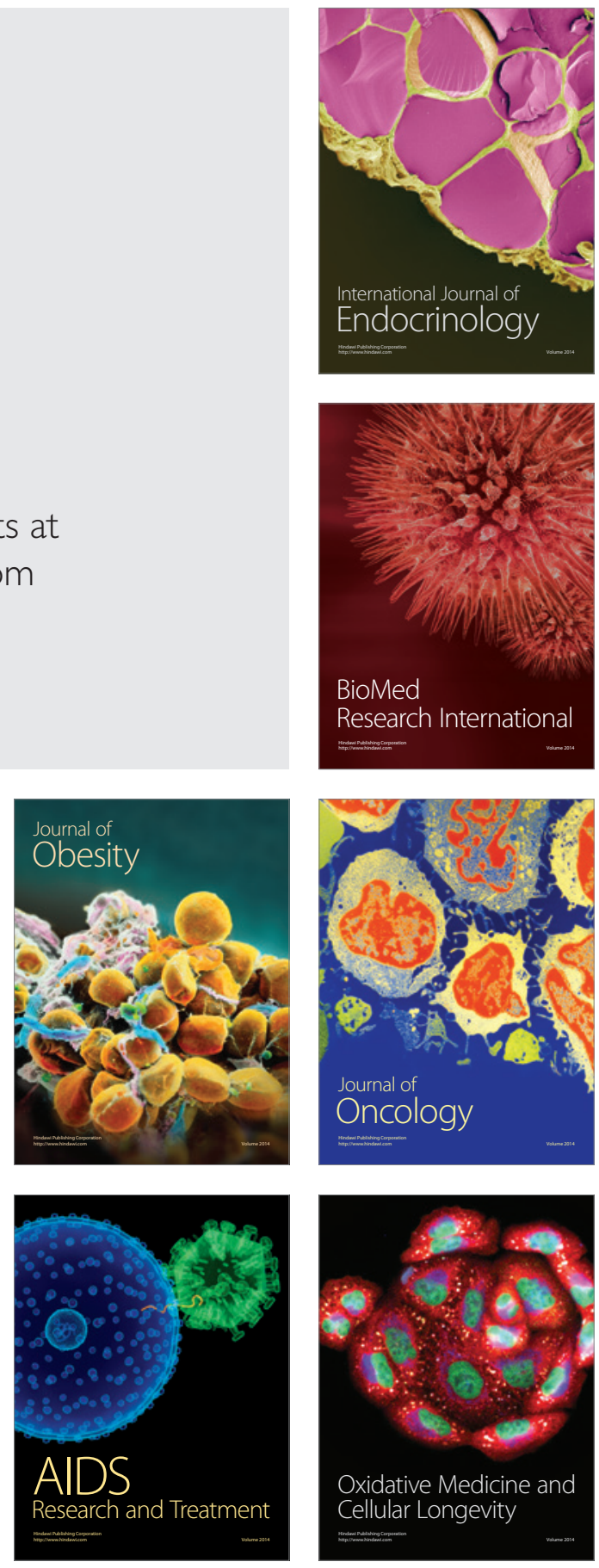